\title{
Efficacy of Sentinel Lymph Node Biopsy in Male Breast Cancer
}

\author{
VINCENT M. CIMMINO, MD, ${ }^{*}$ AMY C. DEGNIM, MD, MICHAEL S. SABEL, MD, KATHLEEN M. DIEHL, MD, \\ LISA A. NEWMAN, MD, AND ALFRED E. CHANG, MD \\ Division of Surgical Oncology, University of Michigan Comprehensive Cancer Center, Ann Arbor, Michigan
}

\begin{abstract}
Background: Sentinel lymph node biopsy (SLNB) is rapidly becoming the standard of care in the treatment of women with early stage breast cancer. Male breast cancer although relatively rare, has typically been treated with mastectomy and axillary lymph node dissection (ALND). Men who develop breast carcinoma have the same risk as their female counterparts of developing the morbidities associated with axillary dissection. SLNB has been championed as a procedure aimed at preventing those morbidities. We recently have evaluated the role of SLNB in the treatment of men with early stage breast cancer.

Methods: Among the 18 men treated at the University of Michigan Medical Center for breast cancer from May 1998 to November 2002, 6 were treated with SLNB.

Results: The mean tumor size was $1.6 \mathrm{~cm}$. The mean patient age was 59.8 years. All of the patients had one or more sentinel lymph nodes identified. Two of the six did not have confirmatory axillary dissection. Three of the six had positive sentinel lymph nodes $(50 \%)$. Only one of the three patients with a positive sentinel node had more nodes positive. One of the six patients had a positive node on frozen section and underwent immediate complete axillary dissection. This patient had no additional positive nodes. No patients in our series had immunohistochemical studies of the lymph nodes.

Conclusions: Men with early stage breast carcinoma may be offered the management option of SLNB since in the hands of experienced surgeons it has a success rate apparently equal to that in their female counterparts.
\end{abstract}

J. Surg. Oncol. 2004;86:74-77. (c) 2004 Wiley-Liss, Inc.

KEY WoRDs: sentinel lymph node biopsy (SLNB); estrogen receptor (ER); axillary lymph node dissection (ALND); ductal carcinoma in situ (DCIS)

\section{INTRODUCTION}

Male breast cancer is a rare entity, accounting for approximately $1 \%$ of all cases of breast carcinoma [1]. For this reason, the data available to evaluate treatment outcomes has not been abundant and much of what we know and what we do in breast cancer is extracted from knowledge of breast carcinoma in women. In an attempt to avoid the morbidity associated with axillary dissection, sentinel lymph node biopsy (SLNB) has become widely implemented in the treatment of female breast cancer [2].

Men are at no less risk for the development of the morbidities associated with axillary dissection. The potential impact for the male, though no more or less than the female, may be somewhat different. For males, since they frequently have vocations in which the physical activity may be more pronounced the impact may be more devastating. With this in mind it is therefore, reasonable to apply the technology of SLNB to males with breast carcinoma.

\section{MATERIALS AND METHODS}

Between May 1998 and November 2002, 18 men were seen with breast cancer at the University of Michigan

\footnotetext{
*Correspondence to: Vincent M. Cimmino, MD, Division of Surgical Oncology, University of Michigan, 3214 Cancer Center, 1500, East Medical Center Drive, Ann Arbor, MI 48109-0932. Fax: (734) 647-9647.

E-mail: vmc@umich.edu

Accepted 27 February 2004

DOI 10.1002/jso.20045

Published online in Wiley InterScience (www.interscience.wiley.com).
} 
Cancer Center. Six men underwent SLNB. Five of the six patients had radioisotope tracer injected followed by lymphoscintigraphy and all six had blue dye injected. The five patients who had the radioisotope injected received a peritumoral injection of $0.3 \mathrm{~m} \mathrm{Ci}$ of Tc-99 $\mathrm{m}$ unfiltered sulfur colloid (Cis US, Bedford, MA) in $4 \mathrm{cc}$ of normal saline. This was given a minimum of $2 \mathrm{hr}$ prior to the surgery. In the operating room $4 \mathrm{cc}$ of isosulfan blue dye (Lymphazurin, Zenith Parenterals, Rosemont, IL) were given as a peritumoral injection in four patients and $3 \mathrm{cc}$ peritumoral with $1 \mathrm{cc}$ intradermal in two patients. The one patient who received lymphazurin blue but no radionucleide was undiagnosed prior to surgery and underwent a biopsy of the mass with frozen section. Following intraoperative confirmation of the carcinoma the patient was injected with lymphazurin blue and the sentinel node was identified.

The SLNB was then performed in all six patients. The gamma probe (Navigator GPS, U.S. Surgical, Norwalk, CT) and blue dye were used to guide us toward the sentinel nodes via the lymphatics. When the background counts in the axilla decreased tenfold and the dye was no longer visible, we terminated our pursuit of the sentinel lymph nodes. One patient had a frozen section of what appeared to be an obvious positive lymph node. After confirmation, he underwent an immediate axillary dissection. All patients had at least one sentinel lymph node identified. No patients underwent immunohistochemical analysis for the presence of cytokeratin.

The sentinel lymph nodes were processed in the following manner: briefly, each sentinel node was measured and entirely cut along its longitudinal axis into sections of 1.5-2 mm thickness. The sections were submitted in formalin for paraffin section histology. Each paraffin block was sectioned at three levels.

\section{RESULTS}

All patients were clinically node-negative preoperatively. The average ages of the patients were 59.8 years (range 51-67). Five patients (83\%) were Caucasian and one was African American (17\%). Five of the six patients had a palpable mass, one of who had a concomitant yellow nipple discharge. One patient presented with a bloody nipple discharge and no palpable mass. Two of the six presented with micro-calcifications. Pathological data on the tumors of these patients can be found in Table I.

Four patients $(66.7 \%)$ had infiltrating ductal carcinoma, one patient $(16.6 \%)$ had extensive DCIS, and one patient $(16.6 \%)$ had intracystic papillary carcinoma with evidence of microinvasion. The estrogen receptor status was only determined in the four patients with invasive ductal carcinoma and they were all positive. The sentinel lymph node was successfully identified in all six patients.
TABLE I. Pathological Data on Tumors in Male Breast Carcinoma Patients who Underwent Sentinel Lymph Node Biopsy Study $(n=6)$

\begin{tabular}{lc}
\hline Characteristic & Number $(\%)$ \\
\hline Tumor type & \\
Invasive ductal carcinoma & $4(66.7)$ \\
Extensive DCIS & $1(16.7)$ \\
Intracystic papillary carcinoma & $1(16.7)$ \\
$\quad$ (microinvasion) & \\
Mean tumor size (cm) & 1.6 (range $0.7-2.8)$ \\
Tumor location & \\
Central & $4(66.7)$ \\
Lower outer & $1(16.7)$ \\
Upper inner & $1(16.7)$ \\
Tumor side & \\
Right & $2(33.3)$ \\
Left & $4(66.7)$ \\
ER & \\
Positive & $4(66.7)$ \\
Negative & 0 \\
$*$ Unknown & $2(33.3)$ \\
Tumor grade & $1(16.7)$ \\
Low & $3(50)$ \\
Intermediate & $1(16.7)$ \\
High & $1(16.7)$ \\
Unknown & \\
Lymphovascular invasion & $2(33.3)$ \\
Present & $4(66.7)$ \\
Absent & \\
DCIS & $3(50)$ \\
Present & $3(50)$ \\
Absent & \\
\hline
\end{tabular}

DCIS, ductal carcinoma in situ; ER, estrogen receptor.

*ER, status not determined for two patients; one with extensive DCIS and one with DCIS and microvasion.

The mean number of sentinel lymph nodes removed was 2.2. Three of the six patients (50\%) had a positive sentinel lymph node and only one of these had additional lymph nodes positive.

Four of the six patients underwent a completion axillary lymph node dissection (ALND). Only one of the four had additional positive lymph nodes [6]. One of the patients underwent frozen section analysis, which was positive. This was followed by a completion axillary dissection in which all nodes were negative. The results of the sentinel node procedure are highlighted in Table II.

To date, there have been very few reports involving the use of SLNB in males. In order to review this topic, we combined our patients with those of two previous reports $[10,15]$. This allowed us to create a pooled database of 23 patients as shown in Tables III and IV.

\section{DISCUSSION}

Male breast carcinoma accounts for approximately $1 \%$ of all breast cancers. The histological status of the axillary nodes remains the single best prognostic factor in 
TABLE II. Sentinel Lymph Node Data $(n=6)$

\begin{tabular}{lc}
\hline Sentinel node detection data $(\mathrm{n}=6)$ & Number $(\%)$ \\
\hline Mean no. of sentinel nodes/patient (range) & $2.3(1-4)$ \\
Sentinel node successfully identified & $6(100)$ \\
Failure & $0(0)$ \\
Patients with positive sentinel lymph nodes* & $3(50)$ \\
Lymphoscintigram & \\
Positive & $5(83.3)$ \\
Negative & $0(0)$ \\
Not done & $1(16.7)$ \\
Lymph node blue only & $1(16.7)$ \\
Lymph node hot only & $0(0)$ \\
Lymph node hot and blue & $5(83.3)$ \\
\hline
\end{tabular}

*All lymph node positive by $\mathrm{H}+\mathrm{E}$.

both male and female patients with breast cancer [3,4]. Traditionally, ALND with histological examination of the axillary lymph nodes is essential in the planning of adjuvant chemotherapy in patients with breast cancer. ALND may be associated with scarring, numbness, loss of range of motion and lymphedema [5].

The standard treatments for breast carcinoma in men have been closely aligned to the treatments in women.

TABLE III. Combination of Data From two Previous Studies $[10,15]$ and the University of Michigan on Tumors in Male Breast Carcinoma Patients who Underwent Sentinel Lymph Node Biopsy $(n=23)$

\begin{tabular}{lc}
\hline Characteristic & Number $(\%)$ \\
\hline Tumor type & \\
$\quad$ Invasive ductal carcinoma & $19(82.6)$ \\
Extensive DCIS & $2(8.70)$ \\
Intracystic papillary carcinoma & $2(8.70)$ \\
$\quad$ (microinvasive) & \\
Mean tumor size (cm.) & $1.4($ range $0.1-3)$ \\
Tumor location & \\
Central & $14(60.9)$ \\
Lower outer & $1(4.4)$ \\
Upper inner & $2(8.7)$ \\
Upper outer & $6(26.1)$ \\
Tumor side & \\
Right & $6(26.1)$ \\
Left & $17(73.9)$ \\
ER & \\
Positive & $19(82.6)$ \\
Negative & 0 \\
*Unknown & $4(17.4)$ \\
Tumor grade & \\
Low & $1(4.4)$ \\
Intermediate & $10(43.5)$ \\
High & $6(26.1)$ \\
Unknown & $6(26.1)$ \\
Lymphovascular invasion & $7(30.4)$ \\
Present & $16(69.6)$ \\
Absent & \\
\hline DCIS, ductal car & \\
\hline
\end{tabular}

DCIS, ductal carcinoma in situ; ER, estrogen receptor. *ER, status not determined for four patients; two with extensive DCIS and microinvasion.
TABLE IV. Sentinel Lymph Node Data From two Previous Studies $[10,15]$ and the University of Michigan $(n=23)$

\begin{tabular}{lc}
\hline Sentinel node detection data $(\mathrm{n}=23)$ & No. $(\%)$ \\
\hline Mean no. of sentinel nodes/patient (range) & $2.67(1-4)$ \\
Sentinel node successfully identified & $22(95.7)$ \\
Failure & $1(4.4)$ \\
Patients with positive sentinel nodes & $9(39.1)$ \\
Positive by frozen section & $3(13.0)$ \\
Positive by H+E only & $6(26.1)$ \\
Positive by deeper sectioning or IHC & $3(13.0)$ \\
Lymphoscintigram & \\
Positive & $17(72.9)$ \\
Negative & $5(21.7)$ \\
Not done & $1(4.4)$ \\
Lymph node blue only & $2(8.7)$ \\
Lymph node hot only & 0 \\
Lymph node hot and blue & $20(87.0)$ \\
\hline
\end{tabular}

IHC, immunohistochemistry.

Ongoing research in the management of early stage breast carcinoma in women is aimed at improving survival and decreasing morbidity. Recently the concept of a minimally-invasive strategy to stage the nodal basin has emerged, altering the traditional staging algorithm based on a level I/II ALND. Studies have shown that sentinel node biopsy (SLNB) can reliably predict the status of the axilla, so that when the sentinel node is clear, the entire axilla can be assumed to be negative and axillary dissection can be avoided [6,7]. The technique has been validated by many studies documenting consistently high success rates and low false negative rates $[8,9]$. To date, there has been no series documenting significant rates of axillary recurrence or lymphedema in women who have undergone SLNB alone.

Men are at risk for the same complications as women with respect to axillary dissection. The potential clinical implications of sentinel node biopsy have different yet equally important concerns related to axillary morbidity. Department of Labor Statistics indicate that men comprise the vast majority of the manual labor force in the United States, accounting for roughly $60 \%$ of all workers in the manufacturing sector and close to $90 \%$ of all workers in industries like mining and construction [10]. This being the case, a significant positive impact on postoperative morbidity and a patient's return to work capability should be realized. We can anticipate that SLNB will reduce direct costs as result of shorter operating time and hospital stay as well as indirect costs because of the reduced postoperative activity that sometimes prevents full use of the arm and therefore, decreased productivity in the workplace.

Although the population-based Surveillance Epidemiology End Result (SEER) Database concluded that males present with significantly $(P<0.001)$ larger primary tumors and more positive axillary nodes than did 
women [16], there are recent reports that demonstrate the remarkable parallels in the histopathology and prognosis of male and female breast cancer [14]. For example, molecular biological studies demonstrate a similar incidence of p53 mutations in the tumors of both men and women [11]. The 10-year survival for men with stage I (T1NOMO) disease is the same as in women (89\%) [12]. SLNB in female patients with $\mathrm{T} 2$ breast tumors appears to have similar success rates and false negative rates when comparing it to those female breast cancer patients with T1 tumors [13]. The role of sentinel lymphadenectomy in males with $\mathrm{T} 2$ tumors may be harder to define because of the low numbers but we may safely assume that its success will mirror the excellent results seen in females. From the pooled data presented in this report of 19 patients with T1 tumors, 8 had positive sentinel lymph nodes $(42 \%)$ detected by either $\mathrm{H}+\mathrm{E}$ or IHC methods. This incidence of positive sentinel nodes is similar to many of the larger series in women.

\section{CONCLUSION}

Sentinel node biopsy in male patients with early stage breast cancers is comparable to its use in women. It seems to be a simple and reliable method for staging the axilla accurately, avoiding the complications of axillary dissection with the secondary benefit of cost reduction.

\section{REFERENCES}

1. Jemal A, Murray T, Samuels A, et al.: Cancer statistics 2003. CA Cancer J Clin 2003;53:5-26.
2. Veronesi U, Pagnelli G, Galimbertic V, et al.: Sentinel node biopsy to avoid axillary dissection in breast cancer with clinical negative nodes. Lancet 1997;349:1864-1867.

3. Fisher B, Ravdin RG, Ausman RK, et al.: Surgical adjuvant chemotherapy in cancer of the breast: Results of a decade of cooperative investigation. Ann Surg 1968;168:337.

4. Borgen PI, Wong Y, Vlams V, et al.: Current management of male breast cancer, a review of 104 cases. Ann Surg 1994;215: 451-459.

5. Petrek JA, Blackwood MM: Axillary dissection: Current practice and technique. Curr Prob Surg 1995;XXXII:309-316.

6. Guilano AE, Kugan DM, Guenther JM, et al.: Lymphatic mapping and sentinel lymphadenectomy for breast cancer. Ann Surg 1994;220:391-401.

7. Veronesi U, Paganelli G, Viale G, et al.: Sentinel node biopsy and axillary dissection in breast cancer: Results in a large series. J Natl Cancer Inst 1999;91:368-373.

8. Krag D, Weaver D, Ashikaga T.: The sentinel node in breast cancer-A multicenter validation study. N Engl J Med 1998;339: 941-946.

9. Giuliano AE, Dale PS, Turner RR, et al.: Improved axillary staging of breast cancer with sentinel lymphadenectomy. Ann Surg 1995;222:394-399.

10. Port ER, Fey JV, Cody HS III, et al.: Sentinel lymph node biopsy in patients with male breast carcinoma. Cancer 2001;91: 319-323.

11. Anelli A, Anelli TFM, Youngson B, et al:: Mutations of the P53 gene in male breast cancer. Cancer 1995;75:2233-2238.

12. Borgen PI: Male breast cancer. Semin Surg Oncol 1991;7: 314-319.

13. Olson JA, Fey J, Winauver J, et al.: Sentinel lymphadectomy accurately predicts nodal status in $\mathrm{T} 2$ breast cancer. J Am Coll Surg 2000;191:593-599.

14. Fitzgibbons PL, Page DL, Weaver D, et al.: Prognostic factors in breast cancer. College of American pathologists consensus statement 1999. Arch Pathol Lab Med 2000;124: 966-978.

15. Mullan MH, Kissin MW: Positive sentinel node biopsy in male breast carcinoma. ANZ J Surg 2001;71:438-440.

16. Anderson WF, Chu KC, Chatterjee N, et al.: Male breast cancer in the SEER database. Am Soc Clin Oncol 2001;(Abstract no): 1691. 